\title{
L'espace des finances publiques. Les aspects fiscaux en géographie politique: le cas de la Suisse
}

\section{Introduction}

Vivant à une époque où les caisses publiques sont presque vides, nous assistons à maintes tentatives de les remplir. Des affirmations générales ainsi que les plaintes proliferent. Il paraît qu'avec les déficits du secteur public les tâches qui lui ont été confiées sont mises en cause. Ainsi, on critique les députés et le peuple d'avoir posé des demandes excessives.

Dans cette situation dramatique, il nous paraît opportun de considérer les finances publiques sous l'angle de différentes échelles spatiales. En géographie politique, si on parle des finances publiques c'est surtout dans l'optique de la crise du secteur public (p. ex. SHORT 1993, p. 85), et par cela on se réfêre essentiellement à l'Etat central. En appliquant une perspective élargie, on devrait viser aussi les niveaux inférieurs de la hiérarchie politique, voire l'Etat régional et l'Etat local. Il pourrait ainsi s'avérer que les critiques formulées à l'égard des finances publiques soient à relativiser.

Cet article n'a pas pour but primordial de confirmer ou infirmer cette modeste hypothèse. Faute de temps, il n'a pas été possible d'analyser de manière approfondie la quantité de données que la statistique fiscale met à notre disposition. Nous aimerions plutôt promouvoir les finances publiques comme un domaine de la géographie politique, vu son traitement extrêmement sommaire jusqu'à présent.

Afin de placer nos réflexions dans un cadre général, nous allons chercher un appui auprès des sciences politiques (sur le système politique) et des sciences économiques (sur les revenus et les dépenses publiques). La Suisse nous servira d'exemple, vu qu'elle connaît un système bien développé des trois niveaux d'Etat.

\section{Le système politique comme agent multiple}

En géographie politique, l'Etat joue un rôle majeur. Vu sous différentes échelles, nous pouvons identifier l'Etat «national» et l'Etat «local» (JOHNSTON 1982). Nous aimerions compléter ce choix par l'Etat "régional" (discuté brièvement par ce même auteur sous les "fédérations»). Il s'agit d'une entité politique à un niveau intermédiaire, que l'on retrouve dans des Etats fédéraux tels que le Canada, les Etats-Unis, l'Australie, l'Allemagne, l'Autriche ou la Suisse. Même si on se demande dans quelle mesure l'Etat régional peut vraiment agir librement
(JOHNSTON 1982, p. 189), il connaît, tout comme les autres niveaux, un système input - output identique à celui esquissé par EASTON (1965). ${ }^{1}$ Pourtant, ce modèle ne reflète pas le rôle du système politique dans la société. Pour connaître ce rôle, il faut faire recours à une idée développée par OFFE en 1973 (citée dans GRAUHAN \& LINDER 1974). Son modèle nous paraît en effet plus proche de la réalité qui est caractérisée par le jeu du pouvoir entre différents acteurs sociaux. OFFE divise le système "société» en trois grands sous-systèmes (Fig. 1): le système normatif ou légitimant (la population), le système politique et le système économique. Evidemment, ces trois sous-systèmes sont en interaction, c'est-à-dire ils sont indissociables. Dans l'optique choisie par OFFE, par contre, il faut faire abstraction des rapports entre les systèmes économique et normatif et se concentrer sur les relations entre la politique et les deux autres sous-systèmes. Le système politique devient ainsi un tampon entre la population et l'économie qui ont des demandes très spécifiques et, en conséquence, appellent à des actions politiques différentes. OFFE parle ainsi de "disjonction organisatrice» fournie par le système politique.

Ce modèle - tout en étant a-spatial - nous permet non seulement de mieux reconnaitre le rôle de la population dans le processus politique (v. à ce propos LEIMGRUBER 1985, 1986) mais aussi de situer le contexte des finances publiques, soit des recettes, soit des dépenses. En effet, dans l'optique de l'Etat redistributeur, ofFE nous démontre que du côté des entrées, l'Etat est fortement lié au système économique (à travers la perception fiscale), tandis que les dépenses sont dirigées en grande partie vers le système normatif(les prestations sociales). Les relations dans le sens inverse sont plutôt immatérielles (les conditions cadre, la légitimité). Ces idées vont donc guider nos réflexions.

\section{Finances publiques: aspects théoriques}

Toute organisation sociale impose des obligations et des responsabilités, mais comporte aussi des droits. Il en découle que l'organisation étatique a besoin des entrées fiscales - fait que Jésus a accentué dans sa réponse aux

Walter Leimgruber, Prof. Dr., Institut de Géographie, Université de Fribourg, Pérolles, 1700 Fribourg 


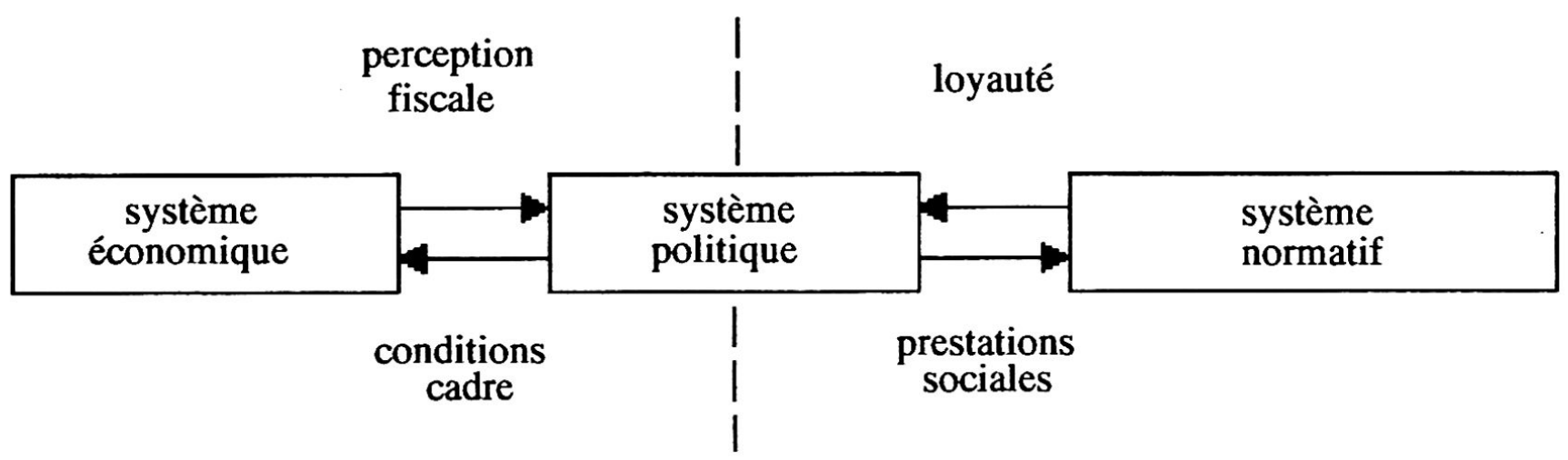

disjonction organisatoire

Fig.1 Le système politique selon OFFE (tiré de GRAUHAN \& LINDNER 1974).

Pharisiens ( «Rendez donc à César ce qui est à César, et à Dieu ce qui est à Dieu», Matthieu 22, 21). Percevoir des impôts est donc une vieille tradition. Pourtant, il nous faut distinguer entre différentes sortes de perceptions. En géographie politique, ce côté entrée des finances publiques n'a guère été étudié, on s'est principalement limité aux dépenses. Dans la théorie des finances publiques, nous trouvons des modèles qui permettent de classifier les deux côtés du bilan. Ainsi, HYMAN (1983) nous fournit un schéma simple que l'on peut retrouver (partiellement du moins) dans les comptes de l'Etat. La problématique des finances publiques est résumée par cet auteur comme suit (p. 348): "A basic problem in government finance is the absence of a quid-pro-quo relationship between government goods and services and a mechanism such as markets and prices to simultaneously ration and finance those goods and services. This difficulty stems from the public good and the redistributive character of many governmental functions. As a result, the distribution of the burden of government finance does not usually bear any systematic relationship to the distribution of government goods and services." Pourtant, ses explications nous permettent de dresser le tableau suivant (Fig. 2):

Les paiements obligatoires sont des recettes d'office: non économiques sont les prestations qui n'ont aucun rapport avec la situation financière des sujets (p.ex. la cote personnelle), tandis que les impôts reflètent leur situation économique. Les impôts directs varient selon le revenu individuel. Les impôts indirects, de leur côté, sont payés par un groupe social particulier (les producteurs, les vendeurs) mais ajoutés au prix de la marchandise ou du service, donc finalement aussi payés par les particuliers, mais selon leur intervention sur le marché. Les prélèvements directs sont des recettes indirectes: l'Etat ne rémunère qu'une fraction de la valeur de marché d'une ressource prélevée (p. ex. la conscription militaire fournit de la main-d'œuvre bon marché pour les tâches de sécurité). Les taxes et émoluments, finalement, sont des entrées pour des services fournis. - Evidemment, ce schéma ne permet pas de classer toutes les entrées (p. ex. les intérêts des placements), il faut donc le compléter par les autres recettes.

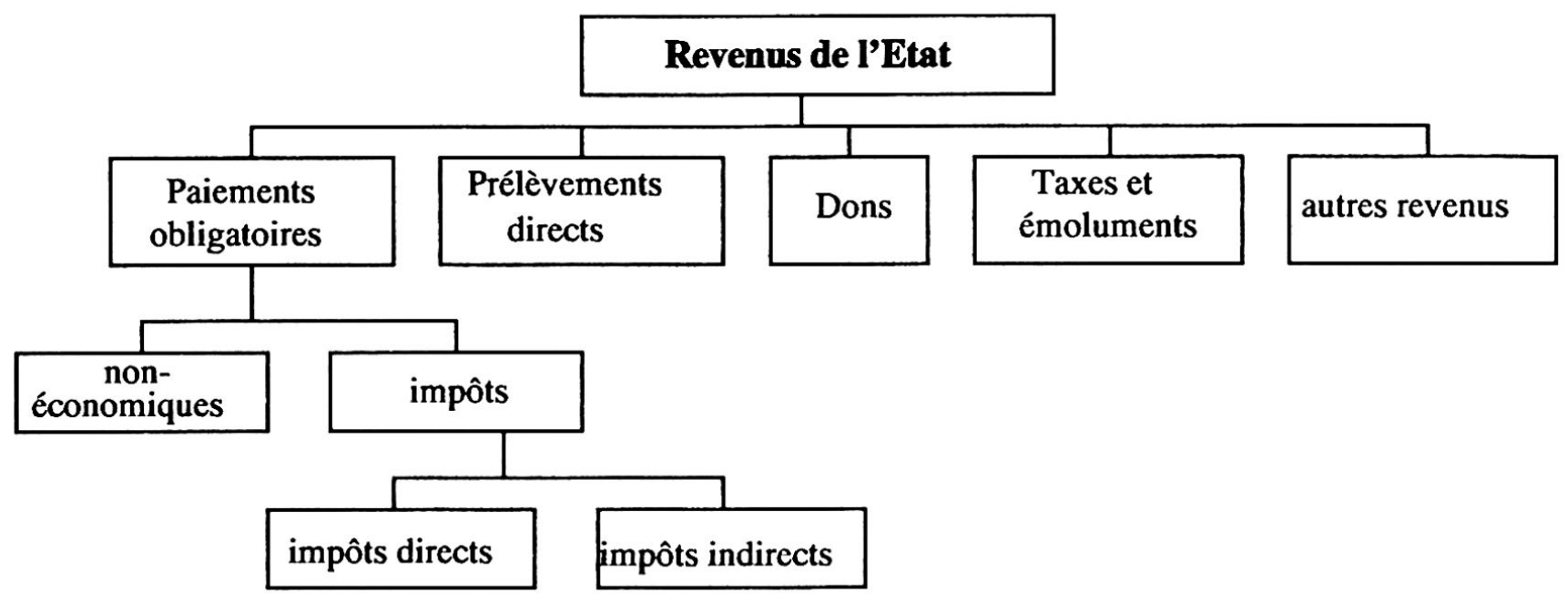

Fig. 2 Schéma des entrées de l'Etat (selon HYMAN 1983, pp. 348-379). 
Du côté des dépenses, nous disposons d'un modèle élaboré par O'CONNOR (1974, p. 16 ss.), qui distingue deux grands volets de dépenses: le côté de la légitimation et celui de l'accumulation (Fig. 3). Du côté de la légitimation se situent les dépenses liées à la sécurité / l'ordre (armée, police; sic! $)^{2}$ et au bien-être social, donc des dépenses qui servent la paix sociale. Dans le volet d'accumulation se placent les dépenses sociales collectives comme l'AVS ainsi que les prestations contre le chômage à court terme (AC) qui s'adressent à la population (le système légitimant); d'autres dépenses, par contre, servent plus ou moins ouvertement le système économique (l'infrastructure physique et les investissements en $\mathrm{R}+\mathrm{D}$ et dans l'éducation), donc l'accumulation du capital à travers les profits. Le rôle disjoncteur de l'Etat entre le sous-système économique et celui normatif ne suit donc pas la grande distinction fournie par les deux volets.

Cette classification paraît assez claire, néanmoins elle simplifie un peu trop. O'CONNOR lui-même est conscient du fait que certaines dépenses peuvent être classées dans les deux volets (1974, p. 18). Ainsi, les routes servent à la fois au transport d'ouvriers (sont donc de l'investissement social), de l'armée et de la police (à classer sous les dépenses sociales). En plus, elle ne tient pas compte de toutes les dépenses. Oủ faudra-t-il placer les coûts de l'administration générale et les frais liés aux finances et impôts? On pourrait les situer dans le volet de la légitimation puisqu'elles reflètent les activités administratives et politiques de l'Etat. Nous avons pourtant jugé opportun de les distribuer pro rata dans les deux volets parce qu'elles servent à la fois la légitimation et l'accumulation. $^{3}$
Malgré leurs légers défauts, ces deux classifications peuvent nous aider à examiner brièvement quelques aspects des finances publiques en Suisse. Dans cet espace limité, nous nous concentrerons sur un choix réduit; néanmoins nous citerons des exemples tirés des trois niveaux d'échelle de l'Etat.

$\mathrm{Si}$ on veut combiner ces deux schémas avec le modèle OFFE, il faut quand même signaler une faiblesse de celuici en ce qui concerne les finances. En effet, il nous suggère que le système politique ne vit que des entrées obtenues du système économique qu'il utilise pour les prestations sociales. En réalité, nous sommes toutes et tous soumis au régime de l'imposition directe (expression de la légitimité), mais au travers les impôts indirects, le système normatif participe aussi aux paiements du système économique. De son côté, l'économie ne se contente pas des conditions cadre mais demande (et reçoit) également des prestations financières (p. ex. les subventions, les primes ou les décharges fiscales pour les nouvelles entreprises). Il faut donc tenir compte de cet élargissement des notions "légitimité» et "conditions cadre» utilisées dans le modèle OFFE.

\section{Les finances publiques de la Confédération}

Un premier aperçu des entrées de la Confédération nous montre que les classifications discutées ci-dessus ne s'appliquent pas automatiquement à une situation quelconque. D'abord, les comptes ne sont pas organisés selon les schémas très simples fournis par HYMAN et O'CONNOR. Il

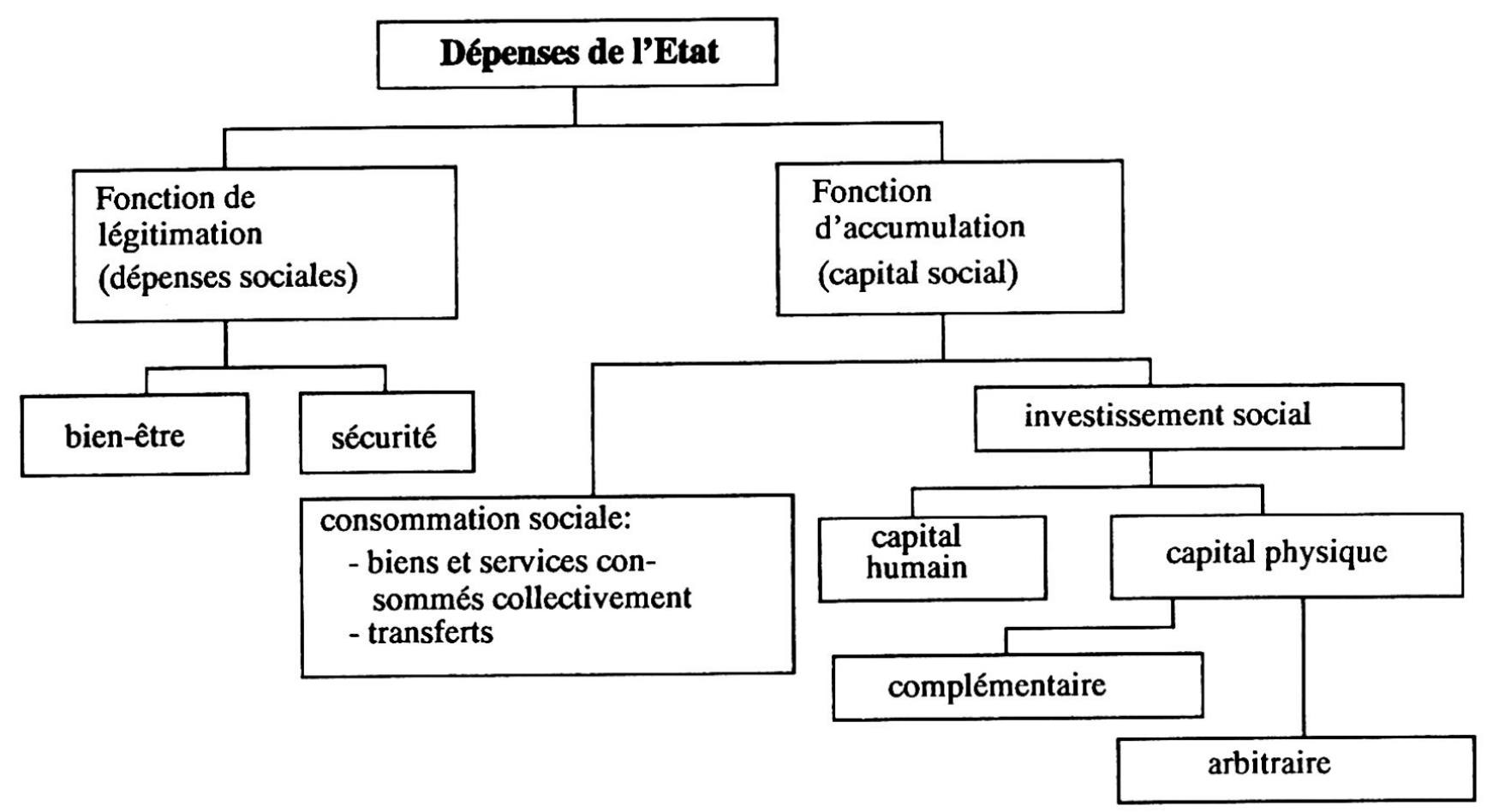

Fig.3 Classification des dépenses de l'Etat (selon O'CONNOR 1974). 
faut tenir compte, par exemple, des contributions financières de la Confédération aux Cantons, des subventions pour les prestations de service public, de la part des cantons aux recettes fédérales, etc. Celles-ci sont propres au système suisse mais ne se découvrent pas aisément dans les comptes. Du côté des entrées, les comptes ne facilitent pas non plus une allocation simple selon les différentes catégories. La taxe non économique, par exemple, est difficile à trouver. La situation est aggravée par le fait que les finances publiques en Suisse se basent sur un nouveau modèle de compte à partir de 1990, ce qui rend la comparaison avec les comptes antérieurs difficile, voire impossible. Il faut donc tenir compte de cette rupture.

Pour une meilleure compréhension des tableaux et figures qui suivront, il convient d'examiner les catégories utilisées par HYMAN et O'CONNOR dans l'optique des comptes helvétiques. Il est effectivement nécessaire d'adapter les deux schémas aux réalités individuelles puisque les deux auteurs écrivent de leur expérience états-unienne.

\section{Côté entrées:}

Paiements obligatoires: les impôts directs et indirects sont facilement reconnaissables dans les comptes; pourtant si les cantons profitent des impôts directs, la Confédération se base surtout sur ceux indirects. Dans ce domaine, il n'y a donc guère de possibilité de comparer les différents niveaux politiques.

Dons: il s'agit ici d'une catégorie négligeable.

Prélèvements directs: les économies dues à la conscription sont difficilement calculables.

Taxes et émoluments: ces recettes sont liées à des prestations, elles sont donc comparables en principe à des prix de marché. Dans notre cas précis elles doivent être considérées comme une catégorie de recettes résiduelles (en incluant les intérêts sur des capitaux).

\section{Côté sorties:}

Dépenses sociales: on y trouve deux catégories: la sécurité (défense, police; on peut y ajouter les pompiers) et le bien-être (la prévoyance sociale et la péréquation financière).

Capital social: nous considérons cette catégorie comme un résidu de tous les autres paiements. Ceci nous semble se défendre vu la division des tâches en Suisse (p. ex. en matière d'éducation et de formation, de dépenses pour le trafic, etc.).

C'est ainsi que nous pouvons établir un premier bilan des recettes et dépenses des collectivités publiques en Suisse pour l'année 1990, date d'entrée en vigueur du nouveau modèle des comptes (Tab. 1):

Ce tableau nous montre l'importance des impôts indirects pour la Confédération du côté recettes ainsi que le rôle des dépenses de légitimation qui sont beaucoup plus élevées qu'au niveau cantonal ou communal. Une confrontation approximative pour la période de 1960 à 1990 nous a montré certains changements dans ce domaine: la part des impôts directs aux recettes a diminué de 61 à $50 \%$ auprès de communes, a oscillé entre 51 et $56 \%$ auprès des cantons, tandis que la Confédération a vu augmenter le pourcentage des impôts directs de $24 \%$ en 1960 à $39 \%$ en 1990 . Nous observons également le niveau élevé des dépenses d'accumulation comparées à celles de légitimation: autour de $75 \%$ auprès des cantons, autour de $81 \%$ dans les communes, et ceci avec une constance remarquable. La Confédération, pour sa part, dépense beaucoup plus pour le volet légitimation (entre 45 et $55 \%)$.

Ces remarques nous permettent de différencier les tâches de l'Etat central par rapport à celles des Etats régionaux et locaux: une bonne partie des dépenses favorisant le système économique (le capital social) est réalisée par les cantons et les communes, tandis que la Confédération porte essentiellement les dépenses sociales pour la paix sociale (pour reprendre les expressions d'O'CONNOR).

\begin{tabular}{|l|c|c|c|}
\hline \multicolumn{1}{|c|}{ Catégorie } & Confédération & Cantons & Communcs \\
\hline Impôts directs & $12^{\prime} 845(39.3 \%)$ & $21^{\prime} 120(53.4 \%)$ & $14^{\prime} 763(50.2)$ \\
\hline Impôts indirects & $15^{\prime} 192(46.5 \%)$ & - & - \\
\hline Autrcs cntrćcs & $4^{\prime} 636(14.2 \%)$ & $18^{\prime} 441(46.6 \%)$ & $145^{\prime} 660(49.8 \%)$ \\
\hline Recettes totales & $32^{\prime} 673(100 \%)$ & $39^{\prime} 561(100 \%)$ & $29^{\prime} 423(100 \%)$ \\
\hline Dépenses de légitimation & $15^{\prime} 881(52.7 \%)$ & $10^{\prime} 896(26.3 \%)$ & $6^{\prime} 399(21.0 \%)$ \\
\hline Dépenses d'accumulation & $14^{\prime} 227(47.3 \%)$ & $30^{\prime} 517(73.7)$ & $23^{\prime} 906(79.0)$ \\
\hline Total de dépenses & $30^{\prime} 108(100 \%)$ & $41^{\prime} 413(100 \%)$ & $30^{\prime} 245(100 \%)$ \\
\hline
\end{tabular}

Tab.1 Entrées et sorties des collectivités suisses, 1990 (en millions de francs). 


\section{Finances publiques et économie}

Il ne faut pourtant pas considérer les finances publiques de façon isolée. Elles sont à voir en rapport avec l'économie entière d'un pays, en ce qui concerne soit les recettes, soit les dépenses. Pour les dernières, il faut tenir compte du fait que l'Etat a de nombreuses tâches (et il continue à en recevoir plus: «des subventions, des systèmes de sécurité sociale, l'aide au développement, la protection de l'environnement»; BOESLER 1983, p. 145). La quote-part des dépenses publiques en est une mesure. On la met normalement en relation avec le PNB nominal, soit comme quote-part nette (dépenses des administrations), soit comme quote-part brute (dépenses des administrations, des entreprises du secteur public et des assurances sociales). Quelle que soit la manière de calculer, force est de constater que la quote-part a sensiblement augmenté depuis 1960 (Tab. 2), même si le maximum a été atteint en $1976(28,2 \%$ pour la quote-part nette, $41,7 \%$ pour la quotepart brute).

\begin{tabular}{|l|c|c|}
\hline Année & $\begin{array}{c}\text { Quote-part } \\
\text { nette (\%) }\end{array}$ & $\begin{array}{c}\text { Quote-part } \\
\text { brute (\%) }\end{array}$ \\
\hline 1960 & 16.9 & 27.4 \\
\hline 1965 & 19.9 & 31.2 \\
\hline 1970 & 21.6 & 32.6 \\
\hline 1975 & 26.3 & 40.8 \\
\hline 1980 & 26.7 & 39.2 \\
\hline 1985 & 26.0 & 39.4 \\
\hline 1990 & 26.4 & inc. \\
\hline
\end{tabular}

Tab.2 Quote-part nette et brute en Suisse, 1960-1990 (en \% des dépenses publiques). Après la révision de la statistique des finances en 1990, on ne dispose pour l'instant pas de données sur les entreprises publiques.

Les recettes de leur part peuvent également se mettre en rapport avec la force économique d'un pays. Parmi les entrées dues aux impôts on peut notamment confronter les charges grevant le bénéfice net et le capital des sociétés anonymes avec le revenu national (par habitant), ce qui nous renseigne sur le rôle différencié de l'Etat régional en matière de politique économique. En effet, la corrélation entre ces deux valeurs est négative et relativement faible $(\mathrm{r}=-0,31)$. Ceci signifie qu'en général les charges grevant les bénéfices et capitaux sont trop élevées dans la plupart des cantons (Fig. 4), que l'instrument de la fiscalité n'est pas partout utilisé pleinement en faveur de la promotion économique (voir à ce propos MOREL 1989, p. 61, qui insiste «sur le fait que la fiscalité n'est pas un élément primordial en matière de promotion économique»). Le canton de Zoug est clairement le mieux placé avec un indice des charges de 57 et du revenu national de 175. Il contraste assez nettement avec les Grisons et Neuchâtel (index des charges de 145 et de 144 , du revenu national de 88 et de 84 respectivement). Nous avons renoncé à inclure dans ce calcul les impôts sur le revenu et la fortune des personnes physiques, mais il est évident qu'ils jouent également un rôle important dans l'économie. Dans ce domaine, c'est également Zoug qui se trouve en tête (index de 55), tandis que le Valais est le canton le moins attractif du point de vue des impôts (index de 151). Une basse fiscalité des personnes physiques favorise les économies et/ou la consommation et rend un canton particulièrement attractif pour certaines catégories de maind'œuvre.

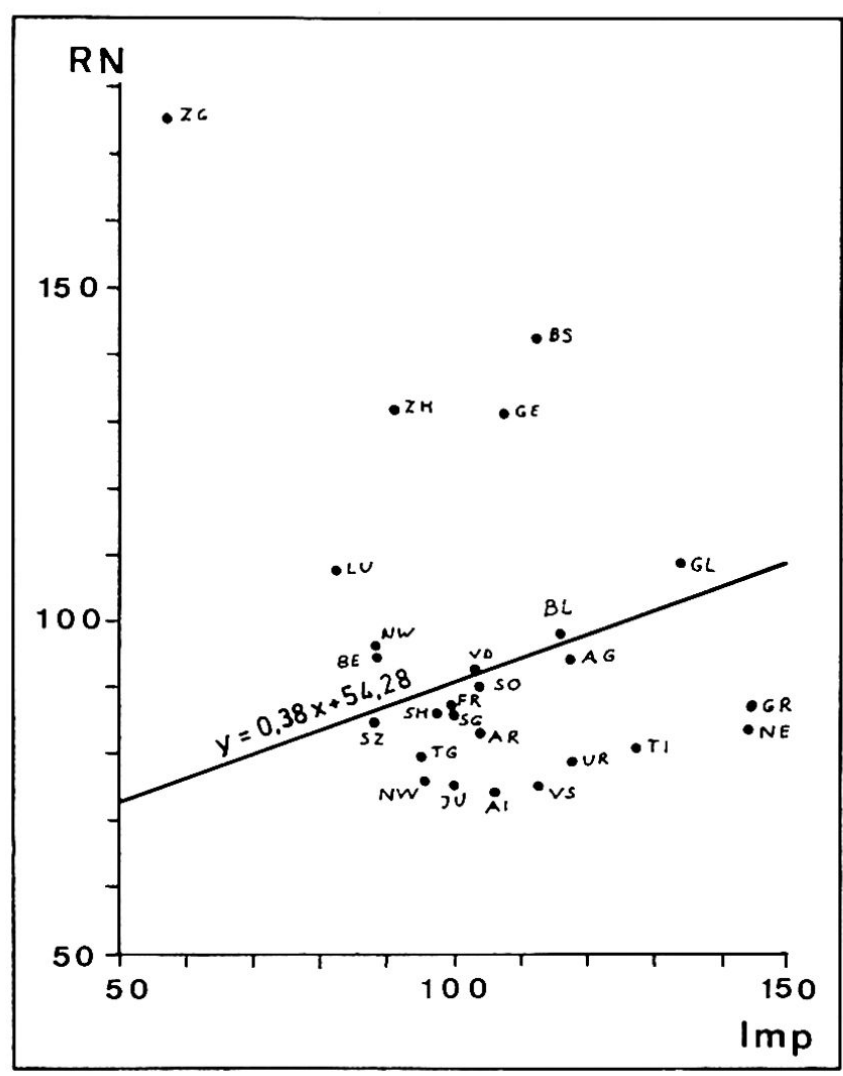

Fig. 4 Revenu national par habitant (RN) et charges grevant le bénéfice net et le capital des sociétés anonymes (Imp) des cantons suisses, 1990 (Index: Suisse = 100).

\section{La crise fiscale de l'Etat}

Les nombreuses tâches de l'Etat ainsi que le fait qu'il devrait être un garant de stabilité à long terme signifie que son comportement financier se distingue de celui d'un entrepreneur. L'Etat doit servir le bien commun, tandis que l'entrepreneur doit constamment veiller à couvrir ses dépenses par des recettes suffisantes pour ne pas finir en faillite. Les deux ne sont donc pas comparables. Pourtant, l'Etat doit aussi veiller à un sain rapport entre les 
entrées et les sorties. Chaque ministre des finances connaît ce problème: demandes croissantes, mais sans frais, si possible. Il y a une certaine inertie dans le système public, qui est décrite comme suit: "Governments are unwilling to levy the taxes needed to raise sufficient money to cover the demanded revenues, because of beliefs that this will harm both capitalist interests and the legitimacy of the system as a whole, as well as their own electoral prospects" (JOHNSTON 1982: 248). On n'augmente pas simplement les prix (c.-à-d. les impôts de tout genre, les taxes et emoluments) pour faire face aux dépenses croissantes - il s'agit d'un problème délicat qui met en cause la légitimité ainsi que les conditions cadres. Le système politique doit donc bien jongler avec sa "disjonction organisatrice» pour ne pas perturber les deux autres sous-systèmes. En l'occurrence, il s'agit de charger plus le partenaire auquel on attribue une moindre importance à long terme ou un moindre potentiel de pression politique. ${ }^{4}$ Dans un système politique hiérarchisé (comme la Suisse), l'Etat central peut déléguer certaines tâches aux niveaux inférieurs pour se décharger, mais ce n'est pas la solution à long terme parce que les possibilités de revenus sont limitées à tout niveau (IBID., p. 249).

Il en résulte une augmentation des déficits et de la dette publique qui varie pourtant d'un niveau à l'autre (Tab. 3). Les soldes des comptes ne sont pas forcément bénéficiaires ou déficitaires aux trois niveaux simultanément. Une bonne gestion ainsi que des coups de fortune, des dépenses réduites ou des entrées inattendues (par exemple les 100 millions de francs des impôts sur la succession encaissés par le canton de Tessin en octobre 1994) conduisent à une différenciation remarquable, surtout au niveau de l'Etat régional et local.

\begin{tabular}{|l|r|r|r|}
\hline Année & $\begin{array}{r}\text { Conféd. } \\
\text { R \% D }\end{array}$ & $\begin{array}{r}\text { Cantons } \\
\text { R \% D }\end{array}$ & $\begin{array}{r}\text { Communes } \\
\text { R \% D }\end{array}$ \\
\hline 1960 & 127.5 & 99.0 & 108.4 \\
\hline 1965 & 100.7 & 93.0 & 95.5 \\
\hline 1970 & 102.7 & 97.4 & 93.7 \\
\hline 1975 & 90.3 & 96.7 & 96.2 \\
\hline 1980 & 93.8 & 99.3 & 102.8 \\
\hline 1985 & 97.0 & 100.9 & 100.6 \\
\hline 1990 & 103.3 & 95.5 & 96.7 \\
\hline
\end{tabular}

Tab. 3 Confrontation des recettes (R) et des dépenses (D) en Suisse, 1960-1990.

Ceci peut être démontré par la confrontation de deux années fiscales diffërentes. Pour un aperçu simple, nous n'avons choisi que peu de chiffres afin de montrer la tendance. Le tableau 4 contient d'un côté le nombre de communes qui en 1987 et en 1991 ont enregistré un excédent de produits dans leurs comptes de fonctionnement, de l'autre côté un sommaire des capitaux et des dettes nettes des communes, toujours groupées par district.

\begin{tabular}{|c|c|c|c|c|c|c|c|c|}
\hline & \multicolumn{2}{|c|}{$\begin{array}{l}\text { Communes } \\
\text { bénéfice }\end{array}$} & \multicolumn{2}{|c|}{$\begin{array}{l}\text { Capital net } \\
\text { (mio.Frs.) }\end{array}$} & \multicolumn{2}{|c|}{$\begin{array}{l}\text { Dette nette } \\
\text { (mio Frs.) }\end{array}$} & \multicolumn{2}{|c|}{$\begin{array}{l}\text { Capital en \% } \\
\text { de la dette }\end{array}$} \\
\hline & 1987 & 1991 & $|1987|$ & 1991 & 1987 & 1991 & 1987 & 1991 \\
\hline & & & 2 & 7 & .1 & 160.8 & 13 & 8.5 \\
\hline & 16 & 14 & $\Delta$ & 0.3 & .1 & 10.7 & 2. & 0.3 \\
\hline & 30 & 30 & 28 & 1.7 & .1 & 21. & 5. & 1.4 \\
\hline $\mathrm{ac}$ & 33 & 27 & 60 & 7.3 & 52.8 & 1.4 & 11.4 & 10.2 \\
\hline & 42 & 0 & .5 & & .8 & 0.4 & 1.3 & 3.0 \\
\hline & 41 & 36 & 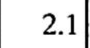 & 1 & 47.7 & 69.8 & 4.4 & 10.2 \\
\hline & 8 & 13 & -- & 0.1 & 38.6 & 37.9 & --1 & 0.3 \\
\hline otal & 217 & 201 & 17.0 & 31.4 & 391.2 & 612.1 & 4.3 & 5.1 \\
\hline
\end{tabular}

Tab. 4 Finances communales dans le canton de Fribourg, 1987 et 1991.

Il s'avère donc qu'en 1987 les $83 \%$ des 260 communes ont bouclé leurs comptes avec un bénéfice, tandis qu'en 1991 ce pourcentage s'est réduit à 78 (sur 256 communes restant après des fusions). Cette dégradation des finances communales a abouti dans un déficit global de 8,2 millions de francs en 1991, tandis qu'en 1987 les communes ont produit un bénéfice de 3,4 millions de francs. Pourtant, la dynamique était inégale. La ville de Fribourg, par exemple, a eu un excédent des entrées en 1987 d'environ 60000 francs, mais un déficit de 6, 5 millions en 1991. Châtel-St-Denis (chef-lieu du district de la Veveyse), pour sa part, a terminé l'année 1987 avec un déficit de 288000 francs, mais 1991 avec un excédent des produits de 10000 francs. Ce phénomène se répète un peu partout dans le canton - mais il existe aussi des communes avec un bénéfice ou un déficit réguliers...

La situation est semblable par rapport aux fortunes. Globalement, les communes fribourgeoises sont fortement endettés. Pourtant, il y en a plusieurs qui disposent d'un capital net (47 en 1987 avec une fortune de 17 millions, 44 en 1991 avec 31 millions). Si l'endettement nominal a augmenté de $64 \%$, le capital a évolué de $85 \%$; il en résulte une amélioration du rapport capital/dette (de 4,3 à 5,1\%). Au niveau local, on semble donc mieux maîtriser la gestion financière, mais il faut surtout considérer les cas individuels. En effet, les comptes varient d'une année à l'autre, et des événements particuliers (fermeture d'une entreprise, par exemple) peuvent avoir des répercussions désastreuses. Rappelons que le canton de Fribourg avait terminé l'année 1991 avec un bénéfice des comptes de fonctionnement de 26,1 millions, mais grâce à un excédent des dépenses d'investissements son déficit arrivait à 67,9 millions.

La conséquence de cette dynamique des finances se reflète dans la dette publique qui a également changé de façon dramatique. Selon le nouveau modèle des finances 

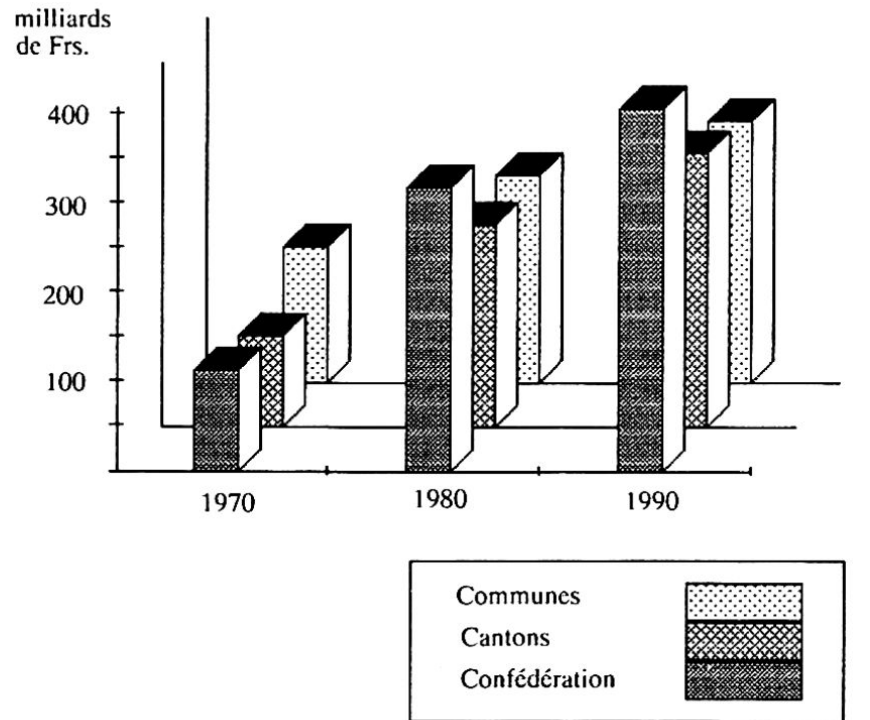

Fig. 5 La dette publique (Confédération, cantons et communes) de 1970 à 1990 (base de calcul: modèle financier 1990). (en vigueur depuis 1990), les trois niveaux participaient de manière variable à la dette publique (Fig. 5). Ainsi, les communes ont réussi à abaisser leur pourcentage de $41,3 \%$ en 1970 à $29,0 \%$ en 1990 , tandis que la Confédération a connu un endettement progressif (de 31,1 à 40,5\%). Les cantons, pour leur part, sont restés relativement stables $(27,6 \%$ en $1970,30,5 \%$ en 1990$)$.

Au niveau spatial concret, la différenciation se montre d'une façon étonnante. En ce qui concerne la charge fiscale totale ${ }^{5}$, c'est la majorité de la population helvétique qui est touchée par une charge excessive (Fig. 6). L'indice de 1990 dépassait la valeur 100 (moyenne Suisse) dans 19 cantons, totalisant 4,27 millions d'habitants $(62,2 \%)$ en 1990 . Les extrêmes étaient Zoug $(56,9)$ et le Valais $(143,0)$.

En ce qui concerne les dépenses, la confrontation peut se faire sous différentes formes. Nous avons choisi deux exemples: l'aspect sécurité (police et pompiers) du côté des dépenses sociales et l'aménagement du territoire/ développement régional du côté accumulation. Ces deux cartes nous informent sur le poids accordé dans les cantons individuels à certains domaines des dépenses.

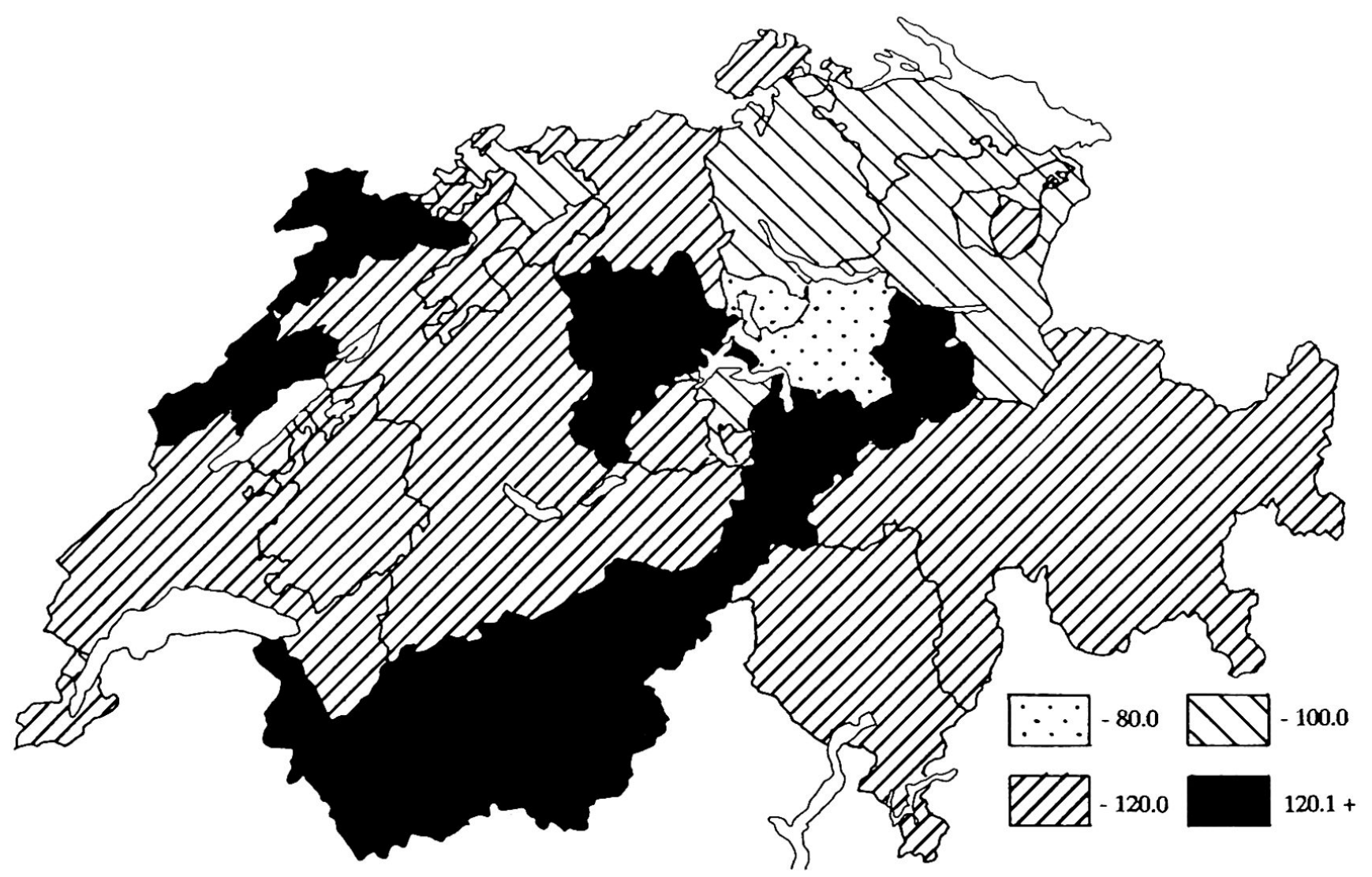

Fig. 6 Charge fiscale, indice global pour 1990 (Suisse $=100$ ). 


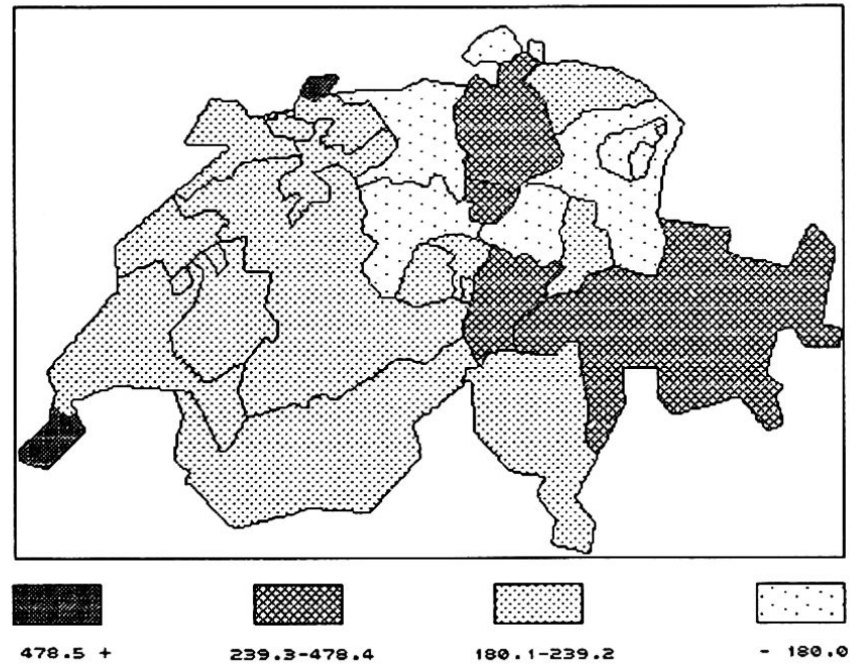

Fig. 7 Dépenses des cantons en 1989 pour la police et les pompiers (francs par habitant).

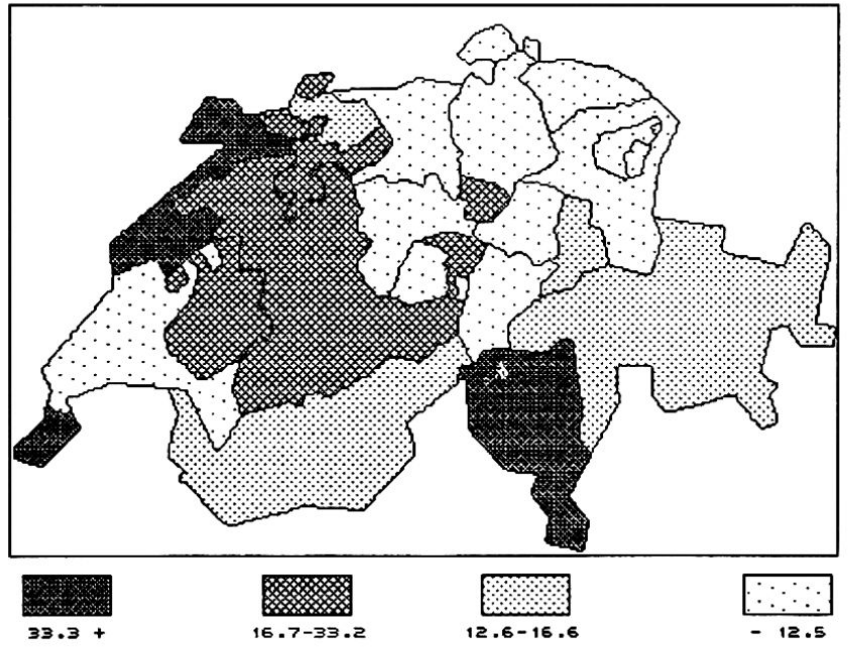

Fig. 8 Dépenses des cantons en 1989 pour l'aménagement du territoire et le développement régional (francs par habitant).
Les frais de police/pompiers (Fig. 7), par exemple, ne sont pas à interpréter selon des priorités politiques des cantons. Il faut plutôt tenir compte de l'organisation interne et des problèmes spécifiques des cantons individuels. Ainsi, Genève et Zurich doivent compter avec des dépenses élevées dans ce domaine à cause des aéroports intercontinentaux et du rôle international des deux villes (soit au niveau politique, soit dans l'économie). $\mathrm{Si}$ on considère les villes de Genève et Zurich, la première réserve $29 \%$ des dépenses de légitimation pour police/ pompiers, la seconde $13 \%$. Des villes comme Fribourg ou Stans s'en sortent avec $5 \%$.

Il en va de même avec les dépenses pour l'aménagement du territoire et le développement régional (Fig. 8). La nécessité ne se fait pas sentir partout de la même manière. Ainsi, Genève, Neuchâtel, le Jura et le Tessin en dépensent beaucoup plus que la Suisse orientale et le canton de Vaud; le Valais et les Grisons font des efforts relativement modestes dans ce domaine comparé à, par exemple, Fribourg, Soleure ou Bâle-Ville, etc.

\section{Perspectives et conclusions}

Le thème des finances publiques n'est pas seulement d'intérêt général (nous payons toutes et tous nos contributions) mais aussi un champ fructueux pour une analyse spatiale approfondie. Ces quelques remarques ont comme but de démontrer l'intérêt de considérer les finances publiques en géographie politique. En premier lieu, nous constatons l'importance de l'organisation in- terne des Etats: l'étude sera différente dans le contexte d'un Etat fédéral que dans un Etat centralisé, vu la hiérarchie de la fiscalité qui reflète la hiérarchie politique. Les différenciations spatiales qui en résultent du côté des revenus sont accompagnées par une différenciation des dépenses. Les premières se basent sur la marche de l'économie, les autres sont en rapport avec les tâches accordées à la collectivité. Une baisse conjoncturelle se traduit donc par une baisse des entrées du système politique (avec des répercussions sur les dépenses: en effet, les demandes de prestations publiques ne finissent pas de s'accroître). En est touché principalement l'Etat central qui reçoit ses entrées du système économique, tandis que l'Etat local dépend pour son revenu beaucoup plus du système normatif. En Suisse, l'Etat régional (les cantons) se trouve entre les deux: ses entrées proviennent d'un côté du système normatif (impôts directs), de l'autre de l'Etat central (contributions de la Confédération). Dans la situation actuelle, il nous parait évident que les années dorées sont passées, mais ceci surtout aux échelles centrale et régionale. Nous avons démontré brièvement que les communes se portent mieux. Ceci est surtout dû au fait qu'elles ne doivent pas porter certaines charges lourdes (en légitimation et en accumulation) qui reviennent aux niveaux supérieurs (sécurité, infrastructures) mais dont elles profitent également. L'hypothèse modeste, formulée au départ, semble donc trouver sa confirmation - ceci au moins pour l'instant. Cette confirmation se base sur la constatation que le contribuable se sent plus responsable au niveau communal (son espace vécu), oủ il peut participer plus facilement aux décisions, qu'au niveau cantonal ou fédéral, où la vue d'ensemble lui échappe. 
1 Ce modèle se résume en quatre termes: entrée (des demandes), prise de décision (dans la boîte noire), sortie (des actions politiques) et rétroaction sur les demandes - le tout dans les confins d'un système spatial. Bien qu'il démontre le fonctionnement du système politique, il a un défaut pour nous géographes: il est a-spatial. C'est JOHNSTON (1979) qui l'a complété en ajoutant cette dimension.

2 Je fais cette remarque explicitement. Selon le modèle OFFE, la légitimation est associée avant tout avec le système normatif (la population). Pourtant, la sécurite et l'ordre sont aussi (et surtout) dans l'intérêt du système économique. Les dépenses liées àl'armée, la police, etc. pourraient donc aussi être classées dans l'investissement social du côté de l'accumulation.

${ }^{3}$ Dans les dépenses de la Confédération (dans le tableau 1), nous avons inclus la rubrique des relations avec l'étranger dans les frais généraux, parce qu'elles servent à la fois la légitimation et l'accumulation.

${ }^{4} \mathrm{C}$ 'est ainsi que dans une période des caisses vides les problèmes d'environnement reçoivent moins d'attention que ceux du marché du travail, même si l'environnement est le grand problème à long terme.

${ }^{5}$ Elle comprend la charge grevant le revenu et la fortune des personnes physiques, la charge grevant le bénéfice et le capital des sociétés anonymes et les impôts sur les véhicules à moteur.
BOESLER, K.-A. (1983): Politische Geographie. Stuttgart. EASTON, D. (1965): A systems' analysis of political life. New York.

GRAUHAN, R. R. et LINDER, W. (1974): Politik der Verstädterung. Frankfurt a. M.

HYMAN, D. N. (1983): Public finance. A contemporary application of theory to policy. Chicago.

JOHNSTON, R.J. (1979): Political, electoral and spatial systems. An essay in political geography. Oxford.

JOHNSTON, R. J. (1982): Geography and the state. An essay in political geography. London \& Basingstoke.

LEIMGRUBER, W. (1985): Politische Willensäußerung und politische Geographie. Dans: UKPIK, Cahiers de I'Institut de Géographie de Fribourg 3, pp. 19-26.

LEIMGRUBER, W. (1986): II ruolo della popolazione in geografia politica e in geografia elettorale. Dans: Carlo Brusa $(\mathrm{Hg}$.$) : Elezioni, territorio, società. Collana dell'lstituto di$ Scienze Geografiche dell'Università di Parma 6: 61-68.

MOREL, F.(1989): Fiscalité, équité et promotion économique. Dans: EEE (éd.), Standortqualität und wirtschaftliche Entwicklung des Kantons Freiburg - Le canton de Fribourg: qualité de lieu d'implantation et développement économique, pp. 51-67. Freiburg/Schweiz.

O'CONNOR, J. (1974): Die Finanzkrise des Staates. Frankfurt a. $\mathrm{M}$.

SHORT, J. R. (1993): An introduction to political geography. 2nd ed. London \& New York: Routledge. 